\title{
Some New Sets and Topologies in Ideal Topological Spaces
}

\author{
R. Manoharan ${ }^{1}$ and P. Thangavelu ${ }^{2}$ \\ ${ }^{1}$ Department of Mathematics, Sathyabama University, Chennai, Tamil Nadu 600119, India \\ ${ }^{2}$ Department of Mathematics, Karunya University, Coimbatore, Tamil Nadu 641114, India
}

Correspondence should be addressed to R. Manoharan; mano_rl@yahoo.co.in

Received 30 July 2013; Accepted 16 September 2013

Academic Editors: Y. Fu and C. Wang

Copyright (C) 2013 R. Manoharan and P. Thangavelu. This is an open access article distributed under the Creative Commons Attribution License, which permits unrestricted use, distribution, and reproduction in any medium, provided the original work is properly cited.

An ideal topological space is a triplet $(X, \tau, \mathfrak{I})$, where $X$ is a nonempty set, $\tau$ is a topology on $X$, and $\mathfrak{I}$ is an ideal of subsets of $X$. In this paper, we introduce $L^{*}$-perfect, $R^{*}$-perfect, and $C^{*}$-perfect sets in ideal spaces and study their properties. We obtained a characterization for compatible ideals via $R^{*}$-perfect sets. Also, we obtain a generalized topology via ideals which is finer than $\tau$ using $R^{*}$-perfect sets on a finite set.

\section{Introduction and Preliminaries}

The contributions of Hamlett and Jankovic [1-4] in ideal topological spaces initiated the generalization of some important properties in general topology via topological ideals. The properties like decomposition of continuity, separation axioms, connectedness, compactness, and resolvability [59] have been generalized using the concept of ideals in topological spaces.

By a space $(X, \tau)$, we mean a topological space $X$ with a topology $\tau$ defined on $X$ on which no separation axioms are assumed unless otherwise explicitly stated. For a given point $x$ in a space $(X, \tau)$, the system of open neighborhoods of $x$ is denoted by $N(x)=\{U \in \tau: x \in U\}$. For a given subset $A$ of a space $(X, \tau), \operatorname{cl}(A)$ and $\operatorname{int}(A)$ are used to denote the closure of $A$ and interior of $A$, respectively, with respect to the topology.

A nonempty collection of subsets of a set $X$ is said to be an ideal on $X$, if it satisfies the following two conditions: (i) If $A \in \mathfrak{I}$ and $B \subseteq A$, then $B \in \mathfrak{I}$; (ii) If $A \in \mathfrak{I}$ and $B \in \mathfrak{I}$, then $A \cup B \in \mathfrak{I}$. An ideal topological space (or ideal space) $(X, \tau, \mathfrak{I})$ means a topological space $(X, \tau)$ with an ideal $\mathfrak{\Im}$ defined on $X$. Let $(X, \tau)$ be a topological space with an ideal $\mathfrak{\Im}$ defined on $X$. Then for any subset $A$ of $X, A^{*}(\mathfrak{I}, \tau)=\{x \in X / A \cap$ $U \notin \mathfrak{I}$ for every $U \in N(x)\}$ is called the local function of $A$ with respect to $\mathfrak{I}$ and $\tau$. If there is no ambiguity, we will write $A^{*}(\mathfrak{I})$ or simply $A^{*}$ for $A^{*}(\mathfrak{I}, \tau)$. Also, $\mathrm{cl}^{*}(A)=A \cup A^{*}$ defines a Kuratowski closure operator for the topology $\tau^{*}(\Im)$ (or simply $\tau^{*}$ ) which is finer than $\tau$. An ideal $\mathfrak{J}$ on a space $(X, \tau)$ is said to be codense ideal if and only if $\tau \cap \mathfrak{J}=\{\emptyset\}$. $X^{*}$ is always a proper subset of $X$. Also, $X=X^{*}$ if and only if the ideal is codense.

Lemma 1 (see [3]). Let $(X, \tau)$ be a space with $\mathfrak{\Im}_{1}$ and $\mathfrak{\Im}_{2}$ being ideals on $X$, and let $A$ and $B$ be two subsets on $X$. Then

(i) $A \subseteq B \Rightarrow A^{*} \subseteq B^{*}$;

(ii) $\mathfrak{I}_{1} \subseteq \mathfrak{\Im}_{2} \Rightarrow A^{*}\left(\mathfrak{\Im}_{2}\right) \subseteq A^{*}\left(\mathfrak{I}_{1}\right)$;

(iii) $A^{*}=\operatorname{cl}\left(A^{*}\right) \subseteq \operatorname{cl}(A)\left(A^{*}\right.$ is a closed subset of $\left.c l(A)\right)$;

(iv) $\left(A^{*}\right)^{*} \subseteq A^{*}$;

(v) $(A \cup B)^{*}=A^{*} \cup B^{*}$;

(vi) $A^{*}-B^{*}=(A-B)^{*}-B^{*} \subseteq(A-B)^{*}$;

(vii) for every $I \in \mathfrak{I},(A \cup I)^{*}=A^{*}=(A-I)^{*}$.

Definition 2 (see [3]). Let $(X, \tau)$ be a space with an ideal $\Im$ on $X$. One says that the topology $\tau$ is compatible with the ideal $\mathfrak{J}$, denoted by $\tau \sim \mathfrak{J}$, if the following holds, for every $A \subseteq X$ : if for every $x \in A$, there exists a $U \in N(x)$ such that $U \cap A \in \mathfrak{I}$, then $A \in \mathfrak{I}$. 
Definition 3. A subset $A$ of an ideal space $(X, \tau, \mathfrak{\Im})$ is said to be

(i) $\tau^{*}$-closed [3] if $A^{*} \subseteq A$,

(ii) ${ }^{*}$-dense-in-itself [10] if $A \subseteq A^{*}$,

(iii) $I$-open [11] if $A \subseteq \operatorname{int}\left(A^{*}\right)$,

(iv) almost $I$-open [12] if $A \subseteq \operatorname{cl}\left(\operatorname{int}\left(A^{*}\right)\right)$,

(v) $I$-dense [7] if $A^{*}=X$,

(vi) almost strong $\beta$ - I-open [13] if $A \subseteq \mathrm{cl}^{*}\left(\operatorname{int}\left(A^{*}\right)\right)$,

(vii) ${ }^{*}$-perfect [10] if $A=A^{*}$,

(viii) regular $I$-closed [14] if $A=(\operatorname{int}(A))^{*}$,

(ix) an $f_{I}$-set [15] if $A \subseteq(\operatorname{int}(A))^{*}$.

Theorem 4 ([3]). Let $(X, \tau)$ be a space with an ideal $\mathfrak{\Im}$ on $X$. Then the following are equivalent.

(i) $\tau \sim \mathfrak{J}$.

(ii) If A has a cover of open sets each of whose intersection with $A$ is $\mathfrak{I}$, then $A$ is in $\mathfrak{I}$.

(iii) For every $A \subseteq X, A \cap A^{*}=\emptyset \Rightarrow A \in \mathfrak{J}$.

(iv) For every $A \subseteq X, A-A^{*} \in \mathfrak{\Im}$.

(v) For every $\tau^{*}$-closed subset $A, A-A^{*} \in \mathfrak{J}$.

(vi) For every $A \subseteq X$, if $A$ contains no nonempty subset $B$ with $B \subseteq B^{*}$, then $A \in \mathfrak{\Im}$.

\section{2. $L^{*}$-Perfect, $R^{*}$-Perfect, and $C^{*}$-Perfect Sets}

In this section, we define three collections of subsets $\mathfrak{\Omega}, \mathfrak{R}$ and $\mathfrak{S}$ in an ideal space and study some of their properties.

Definition 5. Let $(X, \tau, \mathfrak{\Im})$ be an ideal topological space. A subset $A$ of $X$ is said to be

(i) $L^{*}$-perfect if $A-A^{*} \in \mathfrak{\Im}$,

(ii) $R^{*}$-perfect if $A^{*}-A \in \mathfrak{I}$,

(iii) $C^{*}$-perfect if $A$ is both $L^{*}$-perfect and $R^{*}$-perfect.

The collection of $L^{*}$-perfect sets, $R^{*}$-perfect sets, and $C^{*}$ perfect sets in $(X, \tau, \mathfrak{I})$ is denoted by $\mathfrak{R}, \mathfrak{R}$, and $\mathfrak{E}$, respectively.

Remark 6. (i) If $\mathfrak{I}=\{\emptyset\}$, then

$$
\begin{aligned}
\mathbb{Q} & =\left\{A \subseteq X: A \subseteq A^{*}=\operatorname{cl}(A)\right\} \\
& =\text { the collection of all }{ }^{*} \text {-dense-in-itself sets } \\
& =\wp(X),
\end{aligned}
$$

$$
\begin{aligned}
\mathfrak{R} & =\left\{A \subseteq X: A^{*} \subseteq A\right\} \\
& =\text { the collection of all } \tau^{*} \text {-closed sets } \\
& =\left\{A \subseteq X: A=A^{*}=\mathrm{cl}(A)\right\} \\
& =\text { the collection of all closed sets in }(X, \tau) \\
& =\text { the collection of all }{ }^{*} \text {-perfect sets, } \\
\mathfrak{C} & =\left\{A \subseteq X: A^{*} \subseteq A, A \subseteq A^{*}\right\} \\
& =A X: A=A^{*}=\mathrm{cl}(A) \\
& =\text { the collection of all closed sets in }(X, \tau) \\
& =\text { the collection of all }{ }^{*} \text {-perfect sets. }
\end{aligned}
$$

(ii) If $\mathfrak{I}=\mathfrak{P}(X)$, then $\mathfrak{Q}=\mathfrak{T}=\wp(X)$.

(iii) If $\tau \sim \mathfrak{J}$, then $\mathfrak{Q}=\wp(X)$ (by Theorem 4(iv)).

Remark 7. Every ${ }^{*}$-perfect set is both $R^{*}$-perfect and $L^{*}$ perfect (and hence $C^{*}$-perfect). In Proposition 15, we proved that every members of an ideal is both $R^{*}$-perfect and $L^{*}$ perfect (and hence $C^{*}$-perfect). But any nonempty member of an ideal is not a ${ }^{*}$-perfect set. Hence $R^{*}$-perfect and $L^{*}$ perfect sets (and hence $C^{*}$-perfect sets) need not be ${ }^{*}$-perfect.

Proposition 8. If a subset $A$ of an ideal space $(X, \tau, \Im)$ is $C^{*}$ perfect, then $A \Delta A^{*} \in \mathfrak{I}$.

Proof. Since $A$ is both $L^{*}$-perfect and $R^{*}$-perfect, $A-A^{*} \in \mathfrak{J}$ and $A^{*}-A \in \mathfrak{I}$. By the finite additive property of ideals, $\left(A-A^{*}\right) \cup\left(A^{*}-A\right) \in \mathfrak{I}$. Hence $A \Delta A^{*} \in \mathfrak{I}$.

Proposition 9. In an ideal space $(X, \tau, \mathfrak{\Im})$, every $\tau^{*}$-closed set is $R^{*}$-perfect.

Proof. Let $A$ be a $\tau^{*}$-closed set. Therefore, $A^{*} \subseteq A$. Hence $A^{*}-A=\phi=\mathfrak{I}$. Therefore, $A$ is an $R^{*}$-perfect set.

Corollary 10. In an ideal space $(X, \tau, \mathfrak{\Im})$,

(i) $X$ and $\phi$ are $R^{*}$-perfect sets,

(ii) every $\tau$-closed set is $R^{*}$-perfect,

(iii) for any subset $A$ of an ideal topological space $(X, \tau, \Im), c l(A), A^{*}, c l^{*}(A)$ are $R^{*}$-perfect sets,

(iv) every regular-I-closed set is $R^{*}$-perfect.

Proof. The proof follows from Proposition 9.

The following example shows that the converses of Proposition 9 and Corollary 10(iv) are not true.

Example 11. Let $(X, \tau, \Im)$ be an ideal space with $X=\{a, b, c\}$, $\tau=\{\phi, X,\{a\}\}$, and $\mathfrak{I}=\{\phi,\{b\}\}$. The set $\{a, c\}$ is $R^{*}$-perfect set which is not a $\tau^{*}$-closed set and hence not a regular- $I$-closed set.

Proposition 12. If a subset $A$ of an ideal topological space $(X, \tau, \mathfrak{\Im})$ is such that $A \in \mathfrak{\Im}$, then $A$ is $C^{*}$-perfect. 
Proof. Since $A \in \mathfrak{I}, A^{*}=\phi$. Then $A-A^{*}=A \in \mathfrak{I}$ and $A^{*}-A=\phi \in \mathfrak{I}$. Hence $A$ is both an $L^{*}$-perfect and $R^{*}$ perfect set.

Corollary 13. Let $A$ be a subset of an ideal space $(X, \tau, \mathfrak{\Im})$. Consider the following.

(i) If $A \in \mathfrak{\Im}$, then every subset of $A$ is a $C^{*}$-perfect set.

(ii) If $A$ is $R^{*}$-perfect, then $A^{*}-A$ is $C^{*}$-perfect.

(iii) If $A$ is an $L^{*}$-perfect set, then $A-A^{*}$ is a $C^{*}$-perfect set.

(iv) If $A$ is $C^{*}$-perfect, then $A \Delta A^{*}$ is a $C^{*}$-perfect set.

Proof. The proof follows from Proposition 12.

Corollary 14. Let $(X, \tau)$ be a space with an ideal $\mathfrak{J}$ on $X$ such that $\tau \sim \mathfrak{J}$. Then for every $A \subseteq X$,

(i) if $A \cap A^{*}=\phi$, then $A$ is $C^{*}$-perfect;

(ii) $A-A^{*}$ is $C^{*}$-perfect;

(iii) if $A$ contains nonempty subset $B$ with $B \subseteq B^{*}$, then $A$ is $C^{*}$-perfect.

Proof. Follows from Theorem 4 and Proposition 12.

Proposition 15. In an ideal space $(X, \tau, \mathfrak{I})$, every ${ }^{*}$-dense-initself set is an $L^{*}$-perfect set.

Proof. Let $A$ be a ${ }^{*}$-dense-in-itself set of $X$. Then $A \subseteq A^{*}$. Therefore, $A-A^{*}=\phi \in \mathfrak{\Im}$. Hence $A$ is an $L^{*}$-perfect set.

Corollary 16. In an ideal space $(X, \tau, \mathfrak{\Im})$,

(i) every I-dense set is $L^{*}$-perfect,

(ii) every I-open set is $L^{*}$-perfect,

(iii) every almost strong $\beta$-I-open set is $L^{*}$-perfect,

(iv) every almost I-open set is $L^{*}$-perfect,

(v) every regular-I-closed set is $L^{*}$-perfect,

(vi) every $f_{I^{-}}$set is $L^{*}$-perfect.

Proof. Since all the above sets are ${ }^{*}$-dense-in-itself, by Proposition 15 , these sets are $L^{*}$-perfect.

Remark 17. The members of the ideal of an ideal space are $L^{*}$-perfect, but the nonempty members of the ideal are not ${ }^{*}$-dense-in-itself. Therefore, the converses of the above Corollary and Proposition 15 need not to be true.

Proposition 18. In an ideal space $(X, \tau, \mathfrak{\Im})$,

(i) empty set is an $L^{*}$-perfect set,

(ii) $X$ is an $L^{*}$-perfect set if the ideal is codense.

Proof. (i) Since $\phi-\phi^{*}=\phi \in \mathfrak{I}$, the empty set is an $L^{*}$-perfect set. (ii) We know that $X=X^{*}$ if and only if the ideal $\mathfrak{\Im}$ is codense. Then $X-X^{*}=\phi \in \mathfrak{I}$. Hence the result follows.

\section{Main Results}

In this section, we prove that finite union and intersection of $R^{*}$-perfect sets are again $R^{*}$-perfect set. Using these results, we obtain a new topology for the finite topological spaces which is finer than $\tau^{*}$-topology.

In Ideal spaces, usually $A \subset B$ implies $A^{*} \subset B^{*}$. We observe that there are some sets $A$ and $B$ such that $A \subset B$ but $A^{*}=B^{*}$.

Example 19. Let $(X, \tau, \mathfrak{\Im})$ be an ideal space with $X=$ $\{a, b, c, d\}, \tau=\{\phi, X,\{a, c\},\{d\},\{a, c, d\}\}, \mathfrak{J}=\{\phi,\{c\},\{d\}$, $\{c, d\}\}$. Here the sets $A=\{a\}$ and $B=\{a, b\}$ are such that $A \subseteq B$, but $A^{*}=B^{*}=\{a, b, c\}$.

Proposition 20. Let $(X, \tau, \Im)$ be an ideal space. Let $A$ and $B$ be two subsets of $X$ such that $A \subseteq B$ and $A^{*}=B^{*}$; then

(i) $B$ is $R^{*}$-perfect if $A$ is $R^{*}$-perfect;

(ii) $A$ is $L^{*}$-perfect if $B$ is $L^{*}$-perfect.

Proof. (i) Let $A$ be an $R^{*}$-perfect set. Then $A^{*}-A \in \mathfrak{J}$. Now, $B^{*}-B=A^{*}-B \subseteq A^{*}-A$. By heredity property of ideals, $B^{*}-B \in \mathfrak{I}$. Hence $B$ is $R^{*}$-perfect.

(ii) Let $B$ be an $L^{*}$-perfect set. Then $B-B^{*} \in \mathfrak{\Im}$. Now, $A-A^{*}=A-B^{*} \subseteq B-B^{*}$. By heredity property of ideals, $A-A^{*} \in \mathfrak{J}$. Hence $A$ is $L^{*}$-perfect.

Corollary 21. Let $(X, \tau, \Im)$ be an ideal space. Let $A$ and $B$ be two subsets of $X$ such that $A \subseteq B \subseteq c l^{*} A$; then

(i) $B$ is $R^{*}$-perfect if $A$ is $R^{*}$-perfect,

(ii) $A$ is $L^{*}$-perfect if $B$ is $L^{*}$-perfect.

Proof. Since $A \subseteq B \subseteq \mathrm{cl}^{*} A, A^{*} \subseteq B^{*} \subseteq\left(\mathrm{cl}^{*} A\right)^{*}=A^{*}$. Hence $A^{*}=B^{*}$. Therefore, the result follows from Proposition 20 .

Proposition 22. Let $A$ be a subset of an ideal topological space $(X, \tau, \mathfrak{I})$ such that $A$ is $L^{*}$-perfect set and $A \cap A^{*}$ is $R^{*}$-perfect; then both $A$ and $A \cap A^{*}$ are $C^{*}$-perfect.

Proof. Since $A$ is $L^{*}$-perfect, $A-A^{*} \in \mathfrak{I}$. By Lemma 1 (vii), for every $I \in \mathfrak{J},(A \cup I)^{*}=A^{*}=(A-I)^{*}$. Therefore, $(A \cup(A-$ $\left.\left.A^{*}\right)\right)^{*}=A^{*}=\left(A-\left(A-A^{*}\right)\right)^{*}$. This implies $A^{*}=\left(A \cap A^{*}\right)^{*}$. Therefore, we have $A \cap A^{*} \subseteq A$ with $\left(A \cap A^{*}\right)^{*}=A^{*}$. By Proposition 20, $A$ is $R^{*}$-perfect if $A \cap A^{*}$ is $R^{*}$-perfect and $A \cap A^{*}$ is $L^{*}$-perfect if $A$ is $L^{*}$-perfect set. Hence $A$ is $R^{*}$ perfect and $A \cap A^{*}$ is $L^{*}$-perfect.

Proposition 23. If a subset $A$ of an ideal topological space $(X, \tau, \mathfrak{I})$ is $R^{*}$-perfect set and $A^{*}$ is $L^{*}$-perfect, then $A \cap A^{*}$ is $L^{*}$-perfect.

Proof. Since $A$ is $R^{*}$-perfect, $A^{*}-A \in \mathfrak{\Im}$. By Lemma 1(vii), for every $I \in \mathfrak{I},(A \cup I)^{*}=A^{*}=(A-I)^{*}$. Therefore, $\left(A^{*} \cup\left(A^{*}-\right.\right.$ $A))^{*}=A^{* *}=\left(A^{*}-\left(A^{*}-A\right)\right)^{*}$. This implies $A^{* *}=\left(A \cap A^{*}\right)^{*}$. Therefore, we have $A \cap A^{*} \subseteq A^{*}$ with $\left(A \cap A^{*}\right)^{*}=A^{* *}$. By Proposition 20, $A \cap A^{*}$ is $L^{*}$-perfect if $A^{*}$ is $L^{*}$-perfect set. Hence $A \cap A^{*}$ is $L^{*}$-perfect. 
Proposition 24. If $A$ and $B$ are $R^{*}$-perfect sets, then $A \cup B$ is an $R^{*}$-perfect set.

Proof. Let $A$ and $B$ be $R^{*}$-perfect sets. Then $A^{*}-A \in \mathfrak{I}$ and $B^{*}-B \in \mathfrak{J}$. By finite additive property of ideals, $\left(A^{*}-A\right) \cup$ $\left(B^{*}-B\right) \in \mathfrak{I}$. Since $\left(A^{*} \cup B^{*}\right)-(A \cup B) \subseteq\left(A^{*}-A\right) \cup\left(B^{*}-B\right)$, by heredity property $\left(A^{*} \cup B^{*}\right)-(A \cup B) \in \mathfrak{I}$. Hence $(A \cup$ $B)^{*}-(A \cup B) \in \mathfrak{I}$. This proves the result.

Corollary 25. Finite union of $R^{*}$-perfect sets is an $R^{*}$-perfect set.

Proof. The proof follows from Proposition 24.

Proposition 26. If $A$ and $B$ are $L^{*}$-perfect sets, then $A \cup B$ is an $L^{*}$-perfect set.

Proof. Since $A$ and $B$ are $L^{*}$-perfect sets, $A-A^{*} \in \mathfrak{I}$ and $B-$ $B^{*} \in \mathfrak{I}$. Hence by finite additive property of ideals, $\left(A-A^{*}\right) \cup$ $\left(B-B^{*}\right) \in \mathfrak{\Im}$. Since $(A \cup B)-(A \cup B)^{*}=(A \cup B)-\left(A^{*} \cup B^{*}\right) \subseteq$ $\left(A-A^{*}\right) \cup\left(B-B^{*}\right)$, by heredity property $(A \cup B)-(A \cup B)^{*} \in \mathfrak{J}$. This proves that $A \cup B$ is an $L^{*}$-perfect set.

Corollary 27. Finite union of $L^{*}$-perfect sets is an $L^{*}$-perfect sets.

Proof. The proof follows from Proposition 26.

Proposition 28. If $A$ and $B$ are $R^{*}$-perfect sets, then $A \cap B$ is an $R^{*}$-perfect set.

Proof. Suppose that $A$ and $B$ are $R^{*}$-perfect sets. Then $A^{*}-$ $A \in \mathfrak{I}$ and $B^{*}-B \in \mathfrak{J}$. By finite additive property of ideals, $\left(A^{*}-A\right) \cup\left(B^{*}-B\right) \in \mathfrak{I}$. Since $\left(A^{*} \cap B^{*}\right)-(A \cap B) \subseteq\left(A^{*}-\right.$ $A) \cup\left(B^{*}-B\right)$, by heredity property $\left(A^{*} \cap B^{*}\right)-(A \cap B) \in \mathfrak{\Im}$. Also $(A \cap B)^{*}-(A \cap B) \subseteq\left(A^{*} \cap B^{*}\right)-(A \cap B) \in \mathfrak{J}$. This proves the result.

Corollary 29. Finite intersection of $R^{*}$-perfect sets is an $R^{*}$ perfect set.

Proof. The proof follows from Proposition 28.

Proposition 30. Finite union of $C^{*}$-perfect sets is a $C^{*}$-perfect set.

Proof. From Corollaries 27 and 29, finite union of $C^{*}$-perfect sets is a $C^{*}$-perfect set.

Proposition 31. If $(X, \tau, \mathfrak{J})$ is an ideal topological space with $X$ being finite, then the collection $\Re$ is a topology which is finer than the topology of $\tau^{*}$-closed sets.

Proof. By Corollary 10, $X$ and $\phi$ are $R^{*}$-perfect sets. By Corollary 25, finite union of $R^{*}$-perfect sets is an $R^{*}$-perfect set, and by Corollary 29, finite intersection of $R^{*}$-perfect sets is $R^{*}$-perfect. Hence the collection $\Re$ is a topology if $X$ is finite. Also, by Proposition 9 every $\tau^{*}$-closed set is an $R^{*}$ perfect set. Hence the topology $\Re$ is finer than the topology of $\tau^{*}$-closed sets if $X$ is finite.
Proposition 32. In an ideal space $(X, \tau, \mathfrak{I}),\left\{\tau^{*}\right.$-closed sets $\} \cup$ $\mathfrak{I} \subseteq \mathfrak{R}$.

Proof. The proof follows from Propositions 9 and 12.

The following example shows that $\left\{\tau^{*}\right.$-closed sets $\}$ $\mathfrak{I} \neq \mathfrak{R}$

Example 33. Let $(X, \tau, \Im)$ be an ideal space with $X=\{a, b, c\}$, $\tau=\{\emptyset, X,\{a\}\}$, and $\mathfrak{I}=\{\emptyset,\{b\}\}$. The collection of $\tau^{*}$-closed sets is $\{\emptyset, X,\{b\},\{b, c\}\}$ and $\Re=\{\emptyset, X,\{b\},\{b, c\},\{a, c\}\}$.

Now, $\left\{\tau^{*}\right.$-closed sets $\} \cup \mathfrak{J}=\{\emptyset, X,\{b\},\{b, c\}\} \neq \Re$.

Proposition 34. Let $(X, \tau, \mathfrak{J})$ be an ideal space and $A \subseteq X$. The set $A$ is $R^{*}$-perfect if and only if $F \subseteq A^{*}-A$ in $X$ implies that $F \in \mathfrak{I}$.

Proof. Assume that $A$ is an $R^{*}$-perfect set. Then $A^{*}-A \in \mathfrak{I}$. By heredity property of ideals, every set $F \subseteq A^{*}-A$ in $X$ is also in $\mathfrak{I}$. Conversely assume that $F \subseteq A^{*}-A$ in $X$ implies that $F \in \mathfrak{I}$. Since $A^{*}-A$ is a subset of itself, by assumption $A^{*}-A \in \mathfrak{I}$. Hence $A$ is $R^{*}$-perfect.

Proposition 35. Let $(X, \tau, \mathfrak{J})$ be an ideal space and $A \subseteq X$. The set $A$ is $L^{*}$-perfect if and only if $F \subseteq A-A^{*}$ in $X$ implies that $F \in \mathfrak{I}$.

Proof. Assume that $A$ is an $L^{*}$-perfect set. Then $A-A^{*} \in \mathfrak{J}$. By heredity property of ideals, every set $F \subseteq A-A^{*}$ in $X$ is also in $\mathfrak{J}$. Conversely, assume that $F \subseteq A-A^{*}$ in $X$ implies

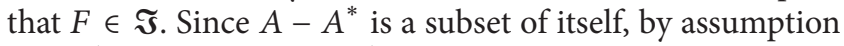
$A-A^{*} \in \mathfrak{I}$. Hence $A$ is $L^{*}$-perfect.

Proposition 36. Let $(X, \tau)$ be a topological space and $A \subseteq X$. Let $\mathfrak{\Im}_{1}$ and $\mathfrak{I}_{2}$ be two ideals on $X$ with $\mathfrak{\Im}_{1} \subseteq \mathfrak{I}_{2}$. Then $A$ is $R^{*}$-perfect with respect to $\Im_{2}$ if it is $R^{*}$-perfect with respect to $\mathfrak{\Im}_{1}$.

Proof. Since $\mathfrak{\Im}_{1} \subseteq \mathfrak{I}_{2}, A^{*}\left(\mathfrak{\Im}_{2}\right) \subseteq A^{*}\left(\mathfrak{I}_{1}\right)$ by Lemma 1(ii) Let $A$ be $R^{*}$-perfect with respect to $\mathfrak{I}_{1}$. Then $A^{*}\left(\mathfrak{I}_{1}\right)-A \in \mathfrak{I}_{1}$. Also, $A^{*}\left(\mathfrak{I}_{2}\right)-A \subseteq A^{*}\left(\mathfrak{I}_{1}\right)-A$. Hence by heredity property of ideals, $A^{*}\left(\mathfrak{I}_{2}\right)-A \in \mathfrak{I}_{1} \subseteq \mathfrak{I}_{2}$. Therefore $A$ is $R^{*}$-perfect with respect to $\Im_{2}$.

Theorem 37. Let $(X, \tau)$ be a space with an ideal $\mathfrak{I}$ on $X$. Then the following are equivalent.

(i) $\tau \sim \mathfrak{J}$

(ii) If $A$ has a cover of open sets each of whose intersection with $A$ is $\mathfrak{J}$, then $A$ is in $\mathfrak{J}$.

(iii) If $A \subseteq X$, then $A \cap A^{*}=\phi \Rightarrow A \in \mathfrak{I}$.

(iv) If $A \subseteq X$, then $A-A^{*} \in \mathfrak{J}$.

(v) If $A \subseteq X$ and $A$ is $R^{*}$-perfect set, then $A \triangle A^{*} \in \mathfrak{J}$.

(vi) For every $\tau^{*}$-closed subset $A, A-A^{*} \in \mathfrak{J}$. 
(vii) For every $A \subseteq X$, if $A$ contains no nonempty subset $B$ with $B \subseteq B^{*}$, then $A \in \mathfrak{\Im}$.

Proof. To prove this theorem, it is enough to prove (iv) $\Rightarrow$ (v) $\Rightarrow$ (vi). Others follow from Theorem 4 . (iv) $\Rightarrow$ (v) follows from Proposition 8. Suppose that $A \Delta A^{*} \in \mathfrak{J}$. Since $A-A^{*} \subseteq$ $A \Delta A^{*}$, by heredity property $A-A^{*} \in \mathfrak{\Im}$. Hence (v) $\Rightarrow$ (vi).

\section{4. $R^{*}$-Topology}

By Corollary 10 and Proposition 28, we observe that the collection $\mathfrak{R}$ satisfies the conditions of being a basis for some topology and it will be called as $R_{c}^{*}(\tau, \mathfrak{\Im})$. We define $R^{*}(\tau, \mathfrak{J})=\left\{A \subseteq X / X-A \in R_{c}^{*}(\tau, \mathfrak{I})\right\}$ on a nonempty set $X$. Clearly, $R^{*}(\tau, \mathfrak{J})$ is a topology if the set $X$ is finite. The members of the collection $R^{*}(\tau, \mathfrak{\Im})$ will be called $R^{*}$ open sets. If there is no confusion about the topology $\tau$ and the ideal $\mathfrak{\Im}$, then we call $R^{*}(\tau, \mathfrak{\Im})$ as $R^{*}$-topology when $X$ is finite.

Definition 38. A subset $A$ of an ideal topological space $(X, \tau, \mathfrak{I})$ is said to be $R^{*}$-closed if it is a complement of an $R^{*}$-open set.

Definition 39. Let $A$ be a subset of an ideal topological space $(X, \tau, \mathfrak{\Im})$. One defines $R^{*}$-interior of the set $A$ as the largest $R^{*}$-open set contained in $A$.One will denote $R^{*}$-interior of a set $A$ by $R^{*}-\operatorname{int}(A)$.

Definition 40. Let $A$ be a subset of an ideal topological space $(X, \tau, \mathfrak{I})$. A point $x \in A$ is said to be an $R^{*}$-interior point of the set $A$ if there exists an $R^{*}$-open set $U$ of $x$ such that $x \in U \subseteq A$.

Definition 41. Let $(X, \tau, \mathfrak{\Im})$ be an ideal space and $x \in X$. One defines $R^{*}$-neighborhood of $x$ as an $R^{*}$-open set containing $x$. One denotes the set of all $R^{*}$-neighborhoods of $x$ by $R^{*}-N(x)$.

Proposition 42. In an ideal space $(X, \tau, \mathfrak{\Im})$, every $\tau^{*}$-open set is an $R^{*}$-open set.

Proof. Let $A$ be a $\tau^{*}$-open set. Therefore, $X-A$ is a $\tau^{*}$-closed set. That implies that $X-A$ is an $R^{*}$-closed set. Hence $A$ is an $R^{*}$-open set.

Corollary 43. The topology $R^{*}(\tau, \mathfrak{I})$ on a finite set $X$ is finer than the topology $\tau^{*}(\tau, \mathfrak{\Im})$.

Proof. The proof follows from Proposition 42.

Corollary 44. For any subset $A$ of an ideal topological space $(X, \tau, \Im)$, int $(A)$ is an $R^{*}$-open set.

Proof. The proof follows from Proposition 42.

Remark 45. (i) Since every open set is an $R^{*}$-open set, every neighborhood $U$ of a point $x \in X$ is an $R^{*}$-neighborhood of $x$. (ii) If $x \in X$ is an interior point of a subset $A$ of $X$, then $x$ is an $R^{*}$-interior point of $A$.

(iii) From (ii), we have $\operatorname{int}(A) \subseteq \operatorname{int}^{*}(A) \subseteq R^{*}$-int $(A)$, where $\operatorname{int}^{*}(A)$ denotes interior of $A$ with respect to the topology $\tau^{*}$.

Theorem 46. Let $A$ and $B$ be subsets of an ideal space $(X, \tau, \mathfrak{\Im})$ with $X$ being finite. Then the following properties hold.

(i) $R^{*}-\operatorname{int}(A)=\cup\left\{U: U \subseteq A\right.$ and $U$ is an $R^{*}$-open set $\}$.

(ii) $R^{*}$-int $(A)$ is the largest $R^{*}$-open set of $X$ contained in A.

(iii) $A$ is $R^{*}$-open if and only if $A=R^{*}-\operatorname{int}(A)$.

(iv) $R^{*}-\operatorname{int}\left(R^{*}-\operatorname{int}(A)\right)=R^{*}-\operatorname{int}(A)$.

(v) If $A \subseteq B$, then $R^{*}-\operatorname{int}(A) \subseteq R^{*}-\operatorname{int}(B)$.

Proof. The proof follows from Definitions 39, 40, and 41.

Definition 47. Let $A$ be a subset of an ideal topological space $(X, \tau, \mathfrak{\Im})$. One defines $R^{*}$-closure of the set $A$ as the smallest $R^{*}$-closed set containing $A$. One will denote $R^{*}$-closure of a set $A$ by $R^{*}-\operatorname{cl}(A)$.

Remark 48. For any subset $A$ of an ideal topological space $(X, \tau, \Im), R^{*}-\mathrm{cl}(A) \subseteq \mathrm{cl}^{*}(A) \subseteq \operatorname{cl}(A)$.

Theorem 49. Let $A$ and $B$ be subsets of an ideal space $(X, \tau, \mathfrak{\Im})$ where $X$ is finite. Then the following properties hold:

(i) $R^{*}-c l(A)=\cap\left\{F: A \subseteq F\right.$ and $F$ is $R^{*}$-closed set $\}$.

(ii) $A$ is $R^{*}$-closed if and only if $A=R^{*}-c l(A)$

(iii) $R^{*}-\operatorname{cl}\left(R^{*}-\operatorname{cl}(A)\right)=R^{*}-\operatorname{cl}(A)$

(iv) If $A \subseteq B$, then $R^{*}-c l(A) \subseteq R^{*}-c l(B)$.

Proof. The proof follows from Definition 47.

Theorem 50. Let $A$ be a subset of an ideal space $(X, \tau, \mathfrak{\Im})$. Then the following properties hold:

(i) $R^{*}-\operatorname{int}(X-A)=X-R^{*}-\operatorname{cl}(A)$;

(ii) $R^{*}-\operatorname{cl}(X-A)=X-R^{*}-\operatorname{int}(A)$.

Proof. The proof follows from Definitions 38, 39, and 47.

\section{Conflict of Interests}

The authors declare that there is no conflict of interests regarding the publication of this paper.

\section{References}

[1] T. R. Hamlett and D. Jankovic, "Ideals in general topology," General Topology and Applications, pp. 115-125, 1988.

[2] T. R. Hamlett and D. Jankovic, "Ideals in topological spaces and the set operatory," Bollettino dell'Unione Matematica Italiana, vol. 7, pp. 863-874, 1990.

[3] D. Jankovic and T. R. Hamlett, "New topologies from old via ideals," The American Mathematical Monthly, vol. 97, pp. 295310, 1990. 
[4] D. Jankovic and T. R. Hamlett, "Compatible extensions of ideals," Bollettino della Unione Matematica Italiana, vol. 7, no. 6, pp. 453-465, 1992.

[5] G. Aslim, A. Caksu Guler, and T. Noiri, "On decompositions of continuity and some weaker forms of continuity via idealization," Acta Mathematica Hungarica, vol. 109, no. 3, pp. 183-190, 2005.

[6] F. G. Arenas, J. Dontchev, and M. L. Puertas, "Idealization of some weak separation axioms," Acta Mathematica Hungarica, vol. 89, no. 1-2, pp. 47-53, 2000.

[7] J. Dontchev, M. Ganster, and D. Rose, "Ideal resolvability," Topology and Its Applications, vol. 93, no. 1, pp. 1-16, 1999.

[8] E. Ekici and T. Noiri, "Connectedness in ideal topological spaces," Novi Sad Journal of Mathematics, vol. 38, no. 2, pp. 6570, 2008.

[9] R. L. Newcomb, Topologies which are compact modulo an ideal [Ph.D. dissertation], University of California at Santa Barbara, 1967.

[10] E. Hayashi, “Topologies defined by local properties," Mathematische Annalen, vol. 156, no. 3, pp. 205-215, 1964.

[11] M. E. Abd El-Monsef, E. F. Lashien, and A. A. Nasef, "On I-open sets and I-continuous functions," Kyungpook Mathematical Journal, vol. 32, no. 1, pp. 21-30, 1992.

[12] M. E. Abd El-Monsef, R. A. Mahmoud, and A. A. Nasef, "Almost-I-openness and almost- $I$ - continuity," Journal of the Egyptian Mathematical Society, vol. 7, no. 2, pp. 191-200, 1999.

[13] E. Hatir, A. Keskin, and T. Noiri, "On new decomposition of continuity via idealization," JP Journal of Geometry \& Topology, vol. 1, no. 3, pp. 53-64, 2003.

[14] A. Keskin, T. Noiri, and Ş. Yüksel, "Idealization of a decomposition theorem," Acta Mathematica Hungarica, vol. 102, no. 4, pp. 269-277, 2004.

[15] A. Keskin, T. Noiri, and S. Yuksel, "fI-sets and decomposition of RIC-continuity," Acta Mathematica Hungarica, vol. 104, no. 4, pp. 307-313, 2004. 


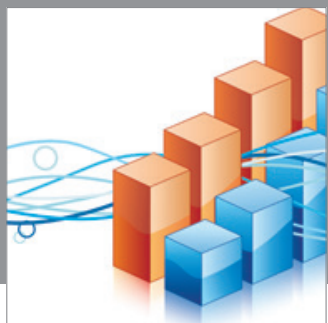

Advances in

Operations Research

mansans

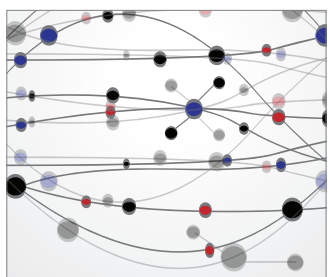

The Scientific World Journal
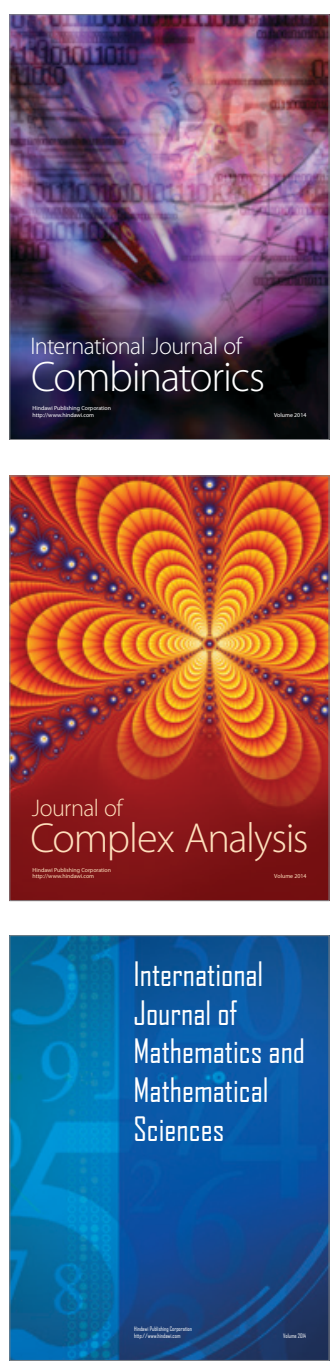
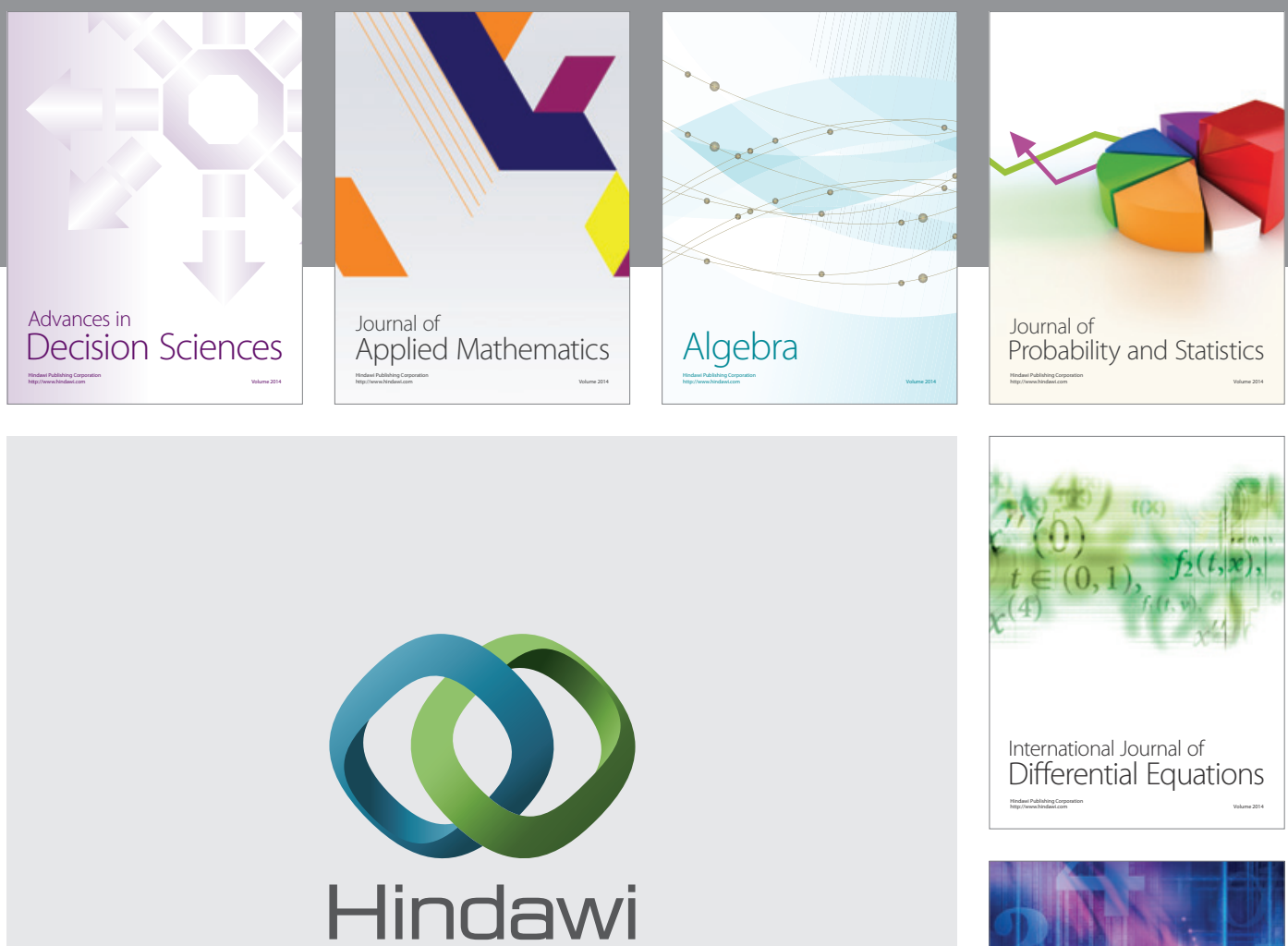

Submit your manuscripts at http://www.hindawi.com
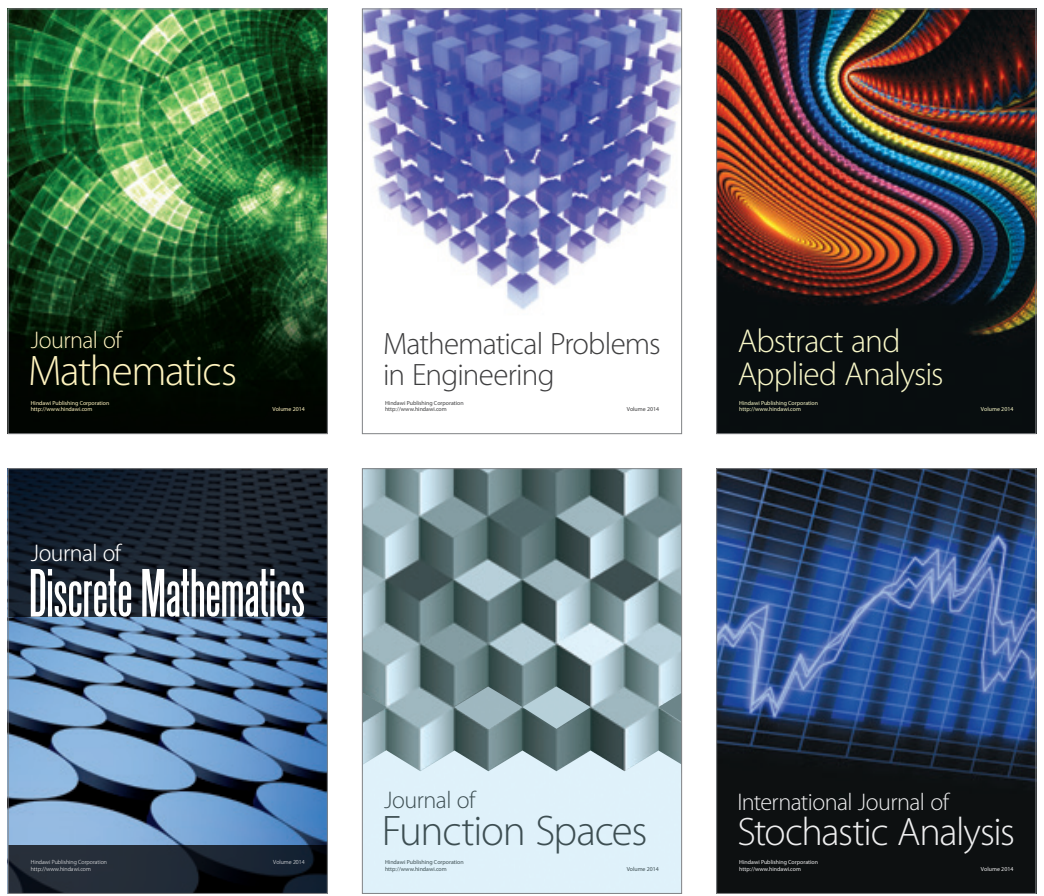

Journal of

Function Spaces

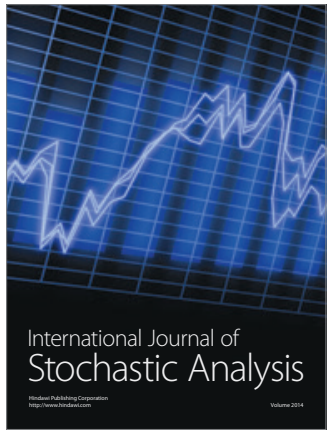

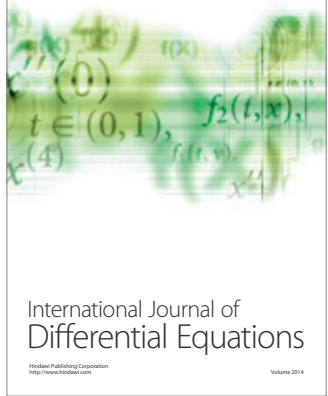
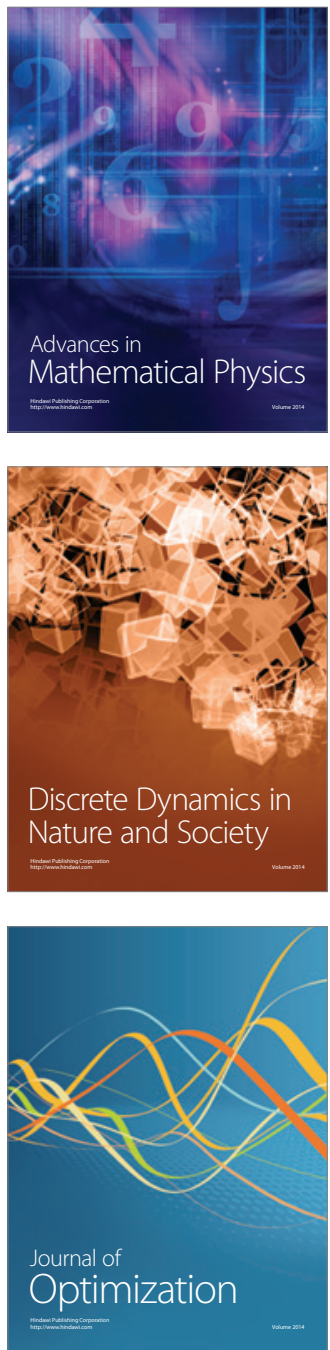